\title{
First-line tyrosine kinase inhibitors in EGFR mutation-positive non-small-cell lung cancer: a network meta-analysis
}

This article was published in the following Dove Medical Press journal:

OncoTargets and Therapy

\author{
Marscha S Holleman ${ }^{1,2}$ \\ Harm van Tinteren ${ }^{3}$ \\ Harry JM Groen ${ }^{4}$ \\ Maiwenn J Al',2 \\ Carin A Uyl-de Groot ${ }^{1,2}$ \\ 'Erasmus School of Health Policy \\ and Management, Erasmus University \\ Rotterdam, Rotterdam, the \\ Netherlands; ${ }^{2}$ Institute for Medical \\ Technology Assessment, Erasmus \\ University Rotterdam, Rotterdam, \\ the Netherlands; ${ }^{3}$ Department of \\ Biometrics, Netherlands Cancer \\ Institute, Amsterdam, the Netherlands; \\ ${ }^{4}$ Department of Pulmonary Diseases, \\ University of Groningen and \\ University Medical Center Groningen, \\ Groningen, the Netherlands
}

Background: EGFR-tyrosine kinase inhibitors (EGFR-TKIs) including afatinib, dacomitinib, erlotinib, gefitinib, and osimertinib have proven efficacy in terms of progression-free survival (PFS) in patients with non-small-cell lung cancer (NSCLC) harboring EGFR mutations. However, an overall view for comparing efficacy and toxicity on a meta-level is lacking. This study compared efficacy and toxicity of first-line treatment with five different EGFR-TKIs by conducting a network meta-analysis (NMA).

Methods: A systematic review was performed, aiming to find eligible literature. Data of PFS, overall survival (OS), objective response rate (ORR), and adverse events were extracted. An NMA based on Bayesian statistics was established to synthesize the efficacy and toxicity of all treatments.

Results: Thirteen randomized controlled trials, including data from 3,539 patients with EGFRmutated NSCLC, were analyzed. Rank probabilities showed that osimertinib had a potentially better efficacy in terms of PFS and OS compared to all other TKIs. For ORR, afatinib and osimertinib showed a trend of superiority compared to the other four TKIs. Furthermore, there was a high risk of diarrhea and rash for patients treated with afatinib or dacomitinib as well as a moderate risk for treatment with erlotinib, gefitinib, and osimertinib.

Conclusion: Our study showed a favorable efficacy of osimertinib in terms of PFS and OS compared to all other EGFR-TKIs in patients with NSCLC harboring activating EGFR mutations. Furthermore, gefitinib, erlotinib, and osimertinib were associated with fewer toxicities compared to the other TKIs. Therefore, osimertinib is indicated as a preferable first-line TKI in patients with activating EGFR-mutated NSCLC.

Keywords: EGFR-TKI, gefitinib, erlotinib, afatinib, osimertinib, network meta-analysis

\section{Introduction}

Lung cancer is the leading cause of cancer-related mortality worldwide. ${ }^{1}$ Of all lung cancer cases, $80-85 \%$ are non-small-cell lung cancers (NSCLC), and the majority of these cases are in advanced or metastatic stage (III or IV) at the time of diagnosis., ${ }^{2,3}$ Among these patients with NSCLC, a substantial number are harboring activating EGFR mutations, ranging from $10 \%$ in Europe to $38.4 \%$ in Asia. ${ }^{4,5}$ During the past years, targeted therapies including tyrosine kinase inhibitors (TKIs) have been developed and have become standard first-line treatment for patients with EGFR mutation-positive NSCLC. ${ }^{6-8}$ Various trials showed higher response rates and improved progression-free survival (PFS) for first-line treatment with afatinib, erlotinib, and gefitinib compared to platinum-based doublet therapy in patients with activating EGFR-mutated (exon 19 deletion or exon 21 L858R mutation) NSCLC. ${ }^{9-18}$ Recently,
Correspondence: Marscha S Holleman Erasmus School of Health Policy and Management, Erasmus University Rotterdam, PO Box 1738, 3000 DR, Rotterdam, the Netherlands Tel +31 104089187 Email holleman@eshpm.eur.nl 
in head-to-head trials, dacomitinib and osimertinib showed a significant longer PFS compared to standard EGFR-TKIs, while dacomitinib, a second-generation EGFR-TKI, had a better efficacy compared to gefitinib, and osimertinib showed a more favorable PFS compared to standard EGFR-TKI (gefitinib or erlotinib). ${ }^{19,20}$ Different EGFR-TKIs are available for the treatment of patients with EGFR mutation-positive NSCLC. However, since sufficient data from head-to-head trials of all these EGFR-TKIs are lacking, evidence of relative efficacy and toxicity of these first-line TKIs is also scarce. Therefore, a network meta-analysis (NMA) was performed to compare the efficacy and toxicity of these TKIs as firstline treatment for patients with EGFR mutation-positive NSCLC. In traditional meta-analyses, the same intervention is compared to the same comparator in all included studies. NMA combines direct comparisons of interventions within randomized controlled trials (RCTs) with indirect comparisons across RCTs in multiple pairwise comparisons across a range of interventions. A greater share of available evidence is produced by using the NMA method compared to traditional meta-analysis. The NMA method enables judicious estimation of the relative treatment effect for comparative effectiveness purposes. ${ }^{21}$ Previously published NMAs did not show significant differences between EGFR-TKIs. ${ }^{22-26} \mathrm{New}$ data for several (new) TKIs are available (ARCHER1050 and FLAURA trials), ${ }^{19,20}$ which may lead to new insights into the relative efficacy and toxicity of the EGFR-TKIs.

This study aimed to compare the efficacy and toxicity of first-line treatment with gefitinib, erlotinib, afatinib, dacomitinib, and osimertinib for patients with activating EGFRmutated (exon 19 deletion or exon 21 L858R mutation) NSCLC by conducting an NMA of all available evidence in the literature.

\section{Materials and methods Search strategy and selection criteria}

An electronic search of the PubMed, EMBASE, and Cochrane Library databases was conducted in order to find eligible studies for the NMA, following PRISMA guidelines. ${ }^{27}$ Eligible studies were Phase IIB/III RCTs that compared the efficacy and toxicity of a single TKI to another TKI or to standard chemotherapy as first-line treatments in patients with stage IIIB/IV NSCLC harboring EGFR mutations and in patients who were not eligible for surgery or radiotherapy. Standard chemotherapy was defined as platinum-based doublet therapy.

Papers published from 1 January, 2010 up to and including 1 November, 2016 were included. Literature was reviewed by two reviewers (MSH and CAUG) and discrepancies were discussed. The selection of studies was based on inclusion and exclusion criteria. Details of the search strategy can be found in Appendix A. Reference lists of published systematic reviews and meta-analyses were checked to ensure that no studies were overlooked. In February 2018, the literature search was manually updated to ensure that no relevant studies were missing, as new trials have been published in the previous 2 years.

\section{Data extraction and quality assessment}

Information on study design, number of participants, patient characteristics, interventions, comparators, objective response rate (ORR) (complete or partial response according to RECIST v1.1), PFS (time from randomization until disease progression according to RECIST v1.1 or death from any cause), overall survival (OS) (time from randomization until death from any cause), and adverse events (AEs) were extracted. Toxicity was scored according to the Common Toxicity Criteria (CTC). ${ }^{28}$ Absolute numbers of AEs were extracted and ORs were calculated. Diarrhea and rash (CTC grade 3 or higher) were included in the analyses of this study because these are the most common TKI-related AEs. Other AEs were not included in the final analysis because they are less impacting and are known to be relatively homogenous across all EGFR-TKIs. ${ }^{29,30}$ Data extraction was verified by the second reviewer (CAUG). For studies with more than one publication, the data were compared between publications. The most updated results were included in this study. Extracted data can be found in Appendix B.

Quality and risk of bias of the RCTs were assessed by using the Cochrane Collaboration's tool for assessing risk of bias. ${ }^{31}$

\section{Statistical analyses}

We performed a Bayesian fixed-effects NMA in WinBUGS 1.4 by using an adapted version of WinBUGS code from Dias et $\mathrm{al}^{32}$ (Appendix C). Due to the limited number of trials in each specific TKI group, a fixed-effects framework was deemed appropriate for the NMA. ${ }^{33-35}$ The outcomes of PFS, OS, ORR, and AEs within trials were linked in a network.

To obtain the HR of treatment a vs b, the following formula was used for all comparisons: $H R_{\mathrm{a}, \mathrm{b}}=\left(e^{(\partial \mathrm{b}-\partial \mathrm{a})}\right)$, and chemotherapy was used as the reference treatment in the network $(\partial$ chemo $=0)$. All other $\partial$ 's were calculated based on direct and indirect evidence from the RCTs. The NMA also enabled us to estimate the probability of being the best treatment and to rank the treatments based on these probabilities. Brooks-Gelman-Rubin diagnostic with WinBUGS was used to assess convergence, which enabled the determination of 
the number of burn-in simulations that should be discarded before calculating the converged results. ${ }^{36}$

The FLAURA trial compared osimertinib with gefitinib or erlotinib. In this trial, no separate HRs of osimertinib vs gefitinib or osimertinib vs erlotinib were reported. Therefore, we assumed that the HRs of PFS and OS were the same for osimertinib vs gefitinib as they were for osimertinib vs erlotinib.

\section{Results}

\section{Identification of studies and study quality}

Electronic search in the databases resulted in 6,182 records, from which 4,664 internal and external duplicates were excluded. Three additional records were included after a manual update of the literature search. After screening the titles and abstracts of the remaining 1,521 records, 66 abstracts and manuscripts were eligible for full-text reading. After this, 53 records were excluded and 13 unique RCTs were included in the analyses. The flow chart is presented in Figure 1.
The patient characteristics of the 13 RCTs are summarized in Table 1. Eight of the 13 RCTs studied gefitinib (NEJ002, WJTOG3405, IPASS, First-SIGNAL, Lux-Lung 6, CTONG0901, ARCHER1050, and FLAURA). ${ }^{9-12,14,19,20,37-41}$ Four RCTs studied erlotinib (OPTIMAL, EURTAC, ENSURE, and CTONG0901), ${ }^{13,15,18,40,42}$ three studied afatinib (Lux-Lung 3, Lux-Lung 6, and Lux-Lung 7), ${ }^{16,17,39,43,44}$ and one trial was included in the analyses for both dacomitinib and osimertinib (the ARCHER1050 and FLAURA study, respectively). ${ }^{19,20}$ Due to the heterogeneous study population of the IPASS and First-SIGNAL trials, we only included the results of the patients with activating EGFR-mutated (exon 19 deletion or exon 21 L858R mutation) NSCLC. A total of 3,539 patients with EGFR mutation-positive NSCLC were available for analyses, 2,691 of whom were randomly assigned to a TKI-arm and 848 of whom received platinumbased doublet therapy. The HRs for PFS and OS, as reported in the trials, are presented in Table 2. All 13 RCTs were classified as having acceptable quality and low risk of bias, according to the Cochrane Collaboration's tool (Appendix D).
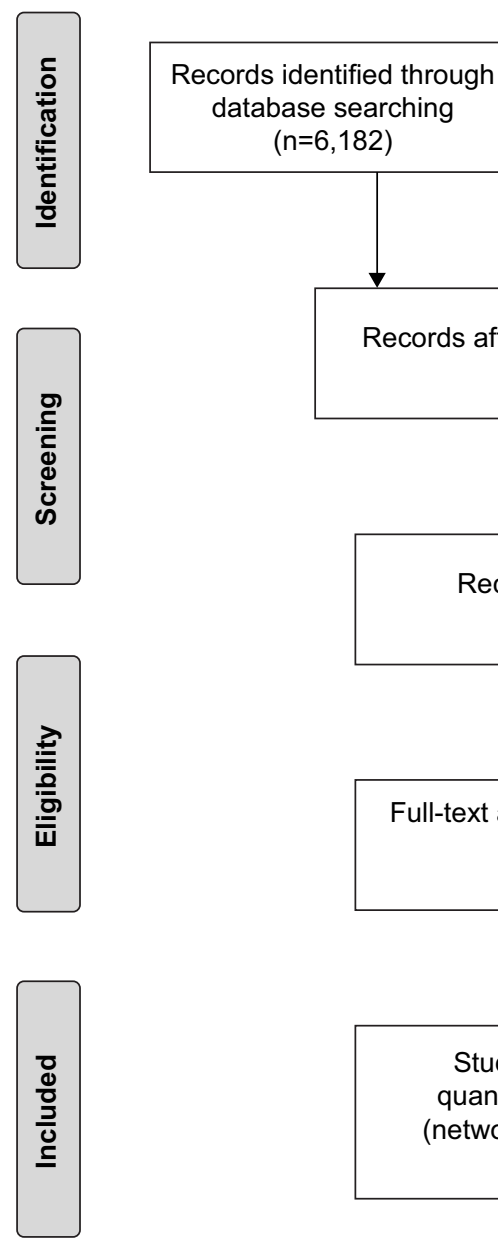

Additional records identified through other sources $(n=3)$

Records after duplicates removed $(n=1,521)$

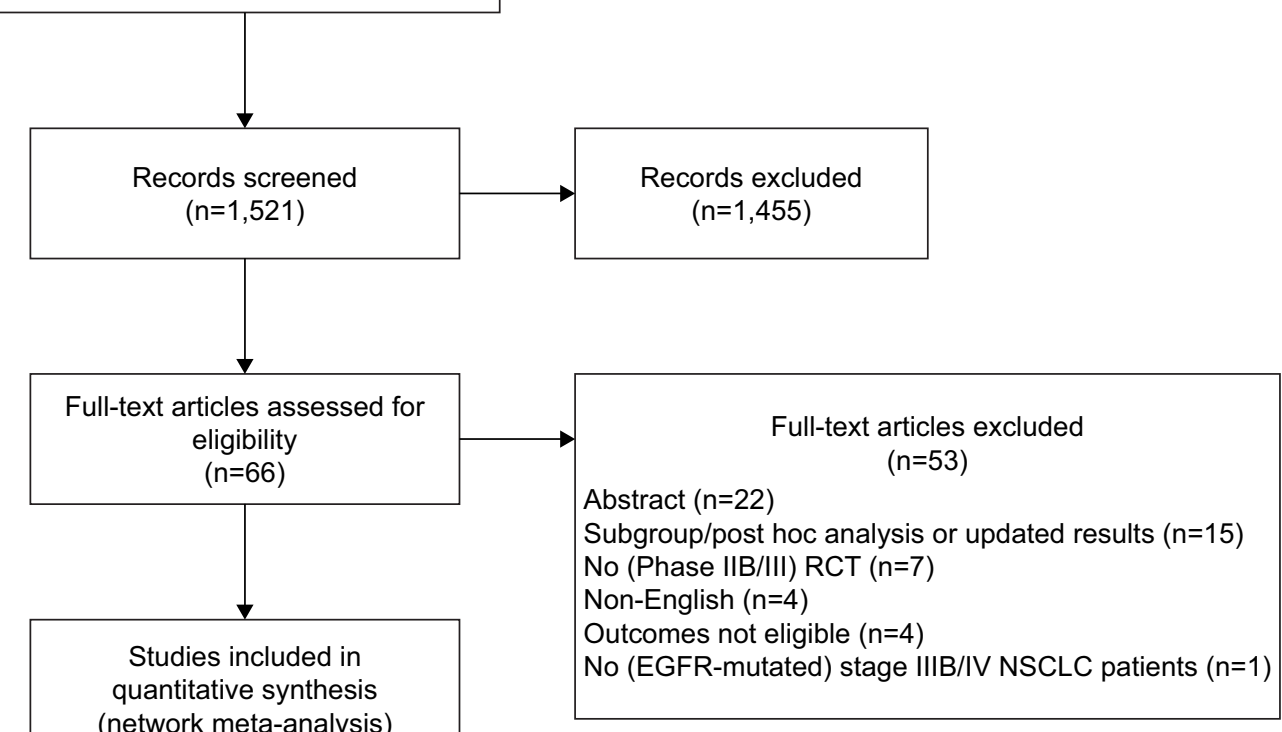
$(n=13)$

Figure I Flow chart of the selection of studies.

Abbreviations: RCT, randomized controlled trial; NSCLC, non-small-cell lung cancer. 
Table I Characteristics of included studies regarding TKIs

\begin{tabular}{|c|c|c|c|c|c|c|c|}
\hline Trial & Treatment & $\begin{array}{l}\text { EGFR } \\
\text { patients }\end{array}$ & Male (\%) & Age & Ethnicity & $\begin{array}{l}\text { Never/previous or } \\
\text { current smoker (\%) }\end{array}$ & $\begin{array}{l}\text { Adenocarcinoma } \\
\text { histology (\%) }\end{array}$ \\
\hline $\mathrm{NEJ002}{ }^{10}$ & $\begin{array}{l}\text { Gefitinib } \\
\text { TC }\end{array}$ & $\begin{array}{l}114 \\
114\end{array}$ & $\begin{array}{l}37 \\
36\end{array}$ & $\begin{array}{l}63.9^{\mathrm{a}} \\
62.6^{\mathrm{a}}\end{array}$ & $\begin{array}{l}\text { Japanese } \\
\text { Japanese }\end{array}$ & $\begin{array}{l}66 / 34 \\
58 / 42\end{array}$ & $\begin{array}{l}90 \\
97\end{array}$ \\
\hline WJTOG3405"I & $\begin{array}{l}\text { Gefitinib } \\
\text { DP }\end{array}$ & $\begin{array}{l}86 \\
86\end{array}$ & $\begin{array}{l}31 \\
30\end{array}$ & $\begin{array}{l}64^{b} \\
64^{b}\end{array}$ & $\begin{array}{l}\text { Japanese } \\
\text { Japanese }\end{array}$ & $\begin{array}{l}71 / 29 \\
66 / 34\end{array}$ & $\begin{array}{l}97 \\
98\end{array}$ \\
\hline $\begin{array}{l}\text { IPASS }{ }^{12} \text { (Fukuoka } \\
\text { et al. 20II) }\end{array}$ & $\begin{array}{l}\text { Gefitinib } \\
\text { TC }\end{array}$ & $\begin{array}{l}132 \\
129\end{array}$ & $\begin{array}{l}21 \\
21\end{array}$ & $\begin{array}{l}57^{\mathrm{b}} \\
57^{\mathrm{b}}\end{array}$ & $\begin{array}{l}\text { Asian } \\
\text { Asian }\end{array}$ & $\begin{array}{l}94 / 6 \\
94 / 6\end{array}$ & $\begin{array}{l}95 \\
97\end{array}$ \\
\hline $\begin{array}{l}\text { First-SIGNAL' } \\
\text { (Han et al. 20I2) }\end{array}$ & $\begin{array}{l}\text { Gefitinib } \\
\text { GP }\end{array}$ & $\begin{array}{l}26 \\
16\end{array}$ & $\begin{array}{l}12 \\
11\end{array}$ & $\begin{array}{l}57^{\mathrm{b}} \\
56.5^{\mathrm{b}}\end{array}$ & $\begin{array}{l}\text { Korean } \\
\text { Korean }\end{array}$ & $\begin{array}{l}\text { N/A } \\
\text { N/A }\end{array}$ & $\begin{array}{l}\text { N/A } \\
\text { N/A }\end{array}$ \\
\hline $\begin{array}{l}\text { OPTIMAL'13 } \\
\text { (Zhou et al. 20II) }\end{array}$ & $\begin{array}{l}\text { Erlotinib } \\
\text { GC }\end{array}$ & $\begin{array}{l}82 \\
72\end{array}$ & $\begin{array}{l}41 \\
40\end{array}$ & $\begin{array}{l}57^{\mathrm{b}} \\
59^{\mathrm{b}}\end{array}$ & $\begin{array}{l}\text { Asian } \\
\text { Asian }\end{array}$ & $\begin{array}{l}72 / 28 \\
69 / 31\end{array}$ & $\begin{array}{l}88 \\
86\end{array}$ \\
\hline $\begin{array}{l}\text { EURTAC }^{15} \text { (Rosell } \\
\text { et al. 20I2) }\end{array}$ & $\begin{array}{l}\text { Erlotinib } \\
\text { CT }\end{array}$ & $\begin{array}{l}86 \\
87\end{array}$ & $\begin{array}{l}33 \\
22\end{array}$ & $\begin{array}{l}65^{\mathrm{b}} \\
65^{\mathrm{b}}\end{array}$ & $\begin{array}{l}\text { European } \\
\text { European }\end{array}$ & $\begin{array}{l}66 / 34 \\
72 / 28\end{array}$ & $\begin{array}{l}95 \\
90\end{array}$ \\
\hline $\begin{array}{l}\text { ENSURE }^{18} \\
\text { (Wu et al. 20I5) }\end{array}$ & $\begin{array}{l}\text { Erlotinib } \\
\text { GC }\end{array}$ & $\begin{array}{l}110 \\
107\end{array}$ & 38 & $\begin{array}{l}57.5^{\mathrm{b}} \\
56^{\mathrm{b}}\end{array}$ & $\begin{array}{l}\text { Asian } \\
\text { Asian }\end{array}$ & $\begin{array}{l}72 / 28 \\
69 / 31\end{array}$ & $\begin{array}{l}95 \\
94\end{array}$ \\
\hline $\begin{array}{l}\text { Lux-Lung } 3^{16} \\
\text { (Sequist et al. 2013) }\end{array}$ & $\begin{array}{l}\text { Afatinib } \\
\text { AP }\end{array}$ & $\begin{array}{l}230 \\
115\end{array}$ & $\begin{array}{l}36 \\
33\end{array}$ & $\begin{array}{l}61.5^{b} \\
61^{b}\end{array}$ & $\begin{array}{l}\text { Global } \\
\text { Global }\end{array}$ & $\begin{array}{l}67 / 33 \\
70 / 30\end{array}$ & $\begin{array}{l}100 \\
100\end{array}$ \\
\hline $\begin{array}{l}\text { Lux-Lung } 6^{17}(\mathrm{Wu} \\
\text { et al. 2014) }\end{array}$ & $\begin{array}{l}\text { Afatinib } \\
\text { GP }\end{array}$ & $\begin{array}{l}242 \\
122\end{array}$ & $\begin{array}{l}36 \\
32\end{array}$ & $\begin{array}{l}58^{\mathrm{b}} \\
58^{\mathrm{b}}\end{array}$ & $\begin{array}{l}\text { Asian } \\
\text { Asian }\end{array}$ & $\begin{array}{l}75 / 25 \\
81 / 19\end{array}$ & $\begin{array}{l}100 \\
100\end{array}$ \\
\hline $\begin{array}{l}\text { Lux-Lung } 7^{39} \text { (Park } \\
\text { et al. 2016) }\end{array}$ & $\begin{array}{l}\text { Afatinib } \\
\text { Gefitinib }\end{array}$ & $\begin{array}{l}160 \\
159 \\
\end{array}$ & $\begin{array}{l}43 \\
33 \\
\end{array}$ & $\begin{array}{l}63^{\mathrm{b}} \\
63^{\mathrm{b}}\end{array}$ & $\begin{array}{l}\text { Global } \\
\text { Global }\end{array}$ & $\begin{array}{l}66 / 34 \\
67 / 33\end{array}$ & $\begin{array}{l}99 \\
99\end{array}$ \\
\hline $\begin{array}{l}\text { CTONG090140 } \\
\text { (Yang et al. 2017) }\end{array}$ & $\begin{array}{l}\text { Erlotinib } \\
\text { Gefitinib }\end{array}$ & $\begin{array}{l}128 \\
128\end{array}$ & $\begin{array}{l}47 \\
46\end{array}$ & c & $\begin{array}{l}\text { N/A } \\
\text { N/A }\end{array}$ & $\begin{array}{l}82 / 18 \\
73 / 27\end{array}$ & $\begin{array}{l}96 \\
96\end{array}$ \\
\hline $\begin{array}{l}\text { ARCHERI05019 } \\
\text { (Wu et al. 2017) }\end{array}$ & $\begin{array}{l}\text { Dacomitinib } \\
\text { Gefitinib }\end{array}$ & $\begin{array}{l}227 \\
225\end{array}$ & $\begin{array}{l}36 \\
44\end{array}$ & $\begin{array}{l}62^{b} \\
61^{b}\end{array}$ & $\begin{array}{l}\text { Global } \\
\text { Global }\end{array}$ & $\begin{array}{l}65 / 26 \\
64 / 36\end{array}$ & $\begin{array}{l}\text { N/A } \\
\text { N/A }\end{array}$ \\
\hline $\begin{array}{l}\text { FLAURA }^{20} \text { (Soria } \\
\text { et al. } 2018)\end{array}$ & $\begin{array}{l}\text { Osimertinib } \\
\text { Standard TKI }\end{array}$ & $\begin{array}{l}279 \\
277\end{array}$ & $\begin{array}{l}36 \\
38\end{array}$ & $\begin{array}{l}64^{b} \\
64^{b}\end{array}$ & $\begin{array}{l}\text { Global } \\
\text { Global }\end{array}$ & $\begin{array}{l}65 / 35 \\
63 / 37\end{array}$ & $\begin{array}{l}99 \\
98\end{array}$ \\
\hline
\end{tabular}

Notes: a Mean; 'bmedian; cin gefitinib arm, 72 patients $(56.3 \%)$ were $\leq 60$ years and 56 patients (43.8\%) were $>60$ years old, and in erlotinib arm, 71 patients $(55.5 \%)$ were $\leq 60$ years and 57 patients $(44.5 \%)$ were $>60$ years.

Abbreviations: TKI, tyrosine kinase inhibitor; EGFR, epidermal growth factor receptor; AP, cisplatin+pemetrexed; CT, chemotherapy (not specific); DP, cisplatin+docetaxel; GC, carboplatin+gemcitabine; GP, cisplatin+gemcitabine; TC, carboplatin+paclitaxel; N/A, not available.

Table 2 HRs for PFS and OS of randomized studies in patients with EGFR-mutated advanced NSCLC treated with TKIs

\begin{tabular}{|c|c|c|c|c|c|}
\hline \multirow[t]{2}{*}{ Trial } & \multirow[t]{2}{*}{ Treatment } & \multirow[t]{2}{*}{ Control } & \multirow{2}{*}{$\begin{array}{l}\text { Primary } \\
\text { end point }\end{array}$} & \multicolumn{2}{|l|}{ HR (95\% Cl) } \\
\hline & & & & PFS & OS \\
\hline NEJ002 $2^{\mathrm{a}, 10}$ (Maemondo et al. 20I0) & Gefitinib & TC & PFS & $0.30(0.22-0.4 \mathrm{I})$ & $0.887(0.634-1.24 I)$ \\
\hline WJTOG3405a,II (Mitsudomi et al. 20I0) & Gefitinib & DP & PFS & $0.489(0.336-0.710)$ & $1.252(0.883-1.775)$ \\
\hline IPASS ${ }^{a, 12}$ (Fukuoka et al. 20II) & Gefitinib & TC & OS & $0.48(0.36-0.64)$ & $1.00(0.76-1.33)$ \\
\hline First-SIGNAL ${ }^{a, 14}$ (Han et al. 20I2) & Gefitinib & GP & OS & $0.544(0.269-1.1)$ & $1.043(0.498-2.182)$ \\
\hline OPTIMAL & Erlotinib & GC & PFS & $0.16(0.10-0.26)$ & I.19 (0.83-I.7I) \\
\hline EURTAC ${ }^{a, 15}$ (Rosell et al. 20I2) & Erlotinib & $\mathrm{CT}$ & PFS & $0.37(0.25-0.54)$ & $1.04(0.65-1.68)$ \\
\hline ENSURE $^{\mathrm{a}, 18}$ (Wu et al. 2015) & Erlotinib & GC & PFS & $0.34(0.22-0.5 \mathrm{I})$ & $0.91(0.63-1.31)$ \\
\hline Lux-Lung 3a,16 (Sequist et al. 20।3) & Afatinib & AP & PFS & $0.58(0.43-0.78)$ & $0.88(0.66-1.17)$ \\
\hline Lux-Lung 6 6,17 (Wu et al. 2014) & Afatinib & GP & PFS & $0.28(0.20-0.39)$ & $0.93(0.72-1.22)$ \\
\hline Lux-Lung $7^{\mathrm{a}, 39}$ (Park et al. 2016) & Afatinib & Gefitinib & PFS, OS & $0.73(0.57-0.95)$ & $0.86(0.66-1.12)$ \\
\hline CTONG090I ${ }^{40}$ (Yang et al. 2017) & Erlotinib & Gefitinib & PFS & $0.96(0.69-1.35)$ & $0.98(0.67-1.42)$ \\
\hline ARCHERI05019 (Wu et al. 2017) & Dacomitinib & Gefitinib & PFS & $0.59(0.47-0.74)$ & $0.76(0.582-0.993)$ \\
\hline FLAURA $^{\mathrm{a}, 20}$ (Soria et al. 2018) & Osimertinib & Standard TKI & PFS & $0.46(0.37-0.57)$ & $0.63(0.45-0.88)$ \\
\hline
\end{tabular}

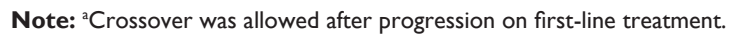

Abbreviations: TKI, tyrosine kinase inhibitor; EGFR, epidermal growth factor receptor; NSCLC, non-small-cell lung cancer; PFS, progression-free survival; OS, overall survival; CT, chemotherapy (not specific); DP, cisplatin+docetaxel; GC, carboplatin+gemcitabine; GP, cisplatin+gemcitabine; N/A, not available; TC, carboplatin+paclitaxel; AP, cisplatin+pemetrexed. 


\section{Network meta-analysis}

Figure 2 shows the complete network, which comprised 13 RCTs that studied a TKI compared to another TKI or chemotherapy in patients with EGFR-mutated NSCLC. We simulated three different chains, which produced 60,000 iterations each. Due to a burn-in period, 30,000 iterations were discarded in each chain; the results were based on a total sample of 90,000 iterations. Brooks-Gelman-Rubin plots showed convergence of the parameters.

Table 3, Figure 3, and Figures S1-S6 in Appendix E present the NMA results for PFS, OS, ORR, and AEs (diarrhea and rash). Osimertinib showed a significantly better PFS and OS compared to all other TKIs. It also had the highest probability of $99 \%$ and $85 \%$, thus showing the longest PFS and OS, respectively, as compared to other TKIs. Dacomitinib also showed a significantly improved PFS compared to gefitinib, erlotinib, and afatinib. Furthermore, afatinib and osimertinib performed best in terms of ORR compared to all other drugs with a probability of $46 \%$ for both drugs. However, the distribution of probabilities of being the best did not differ significantly on ORR (Figure 3).

Diarrhea occurred significantly more often in patients treated with afatinib or dacomitinib. Gefitinib, erlotinib, and osimertinib showed a mild risk of diarrhea and chemotherapy had a low risk, with probabilities of being the best for diarrhea, with $7 \%, 6 \%, 15 \%$, and $72 \%$, respectively. Regarding rash, occurrence was high among patients treated with afatinib or dacomitinib and moderate among patients treated with gefitinib, erlotinib, or osimertinib. The risk of rash was low for chemotherapy with $99 \%$ probability of being the best treatment.

\section{Discussion}

In patients with EGFR-mutated NSCLC, TKIs have shown superior efficacy compared to platinum-based doublet therapy. ${ }^{10-18}$ Now that we have at least five different EGFRTKIs, the relative efficacy and toxicity of these TKIs become important to help physicians choose the optimal drug for treatment. In contrast to meta-analysis, which only estimates the relative effect of the same interventions with the same comparators, an NMA combines direct evidence within RCTs with indirect evidence across RCTs to estimate the relative effect of multiple pairwise comparisons. In this way, the relative efficacy of a whole set of treatments for a disease can be synthesized..$^{21}$ Previous NMAs tried to provide relative evidence on the efficacy of EGFR-TKIs by using only three or four TKIs, or by including both first- and second-line TKIs in the network. These studies did not show significant

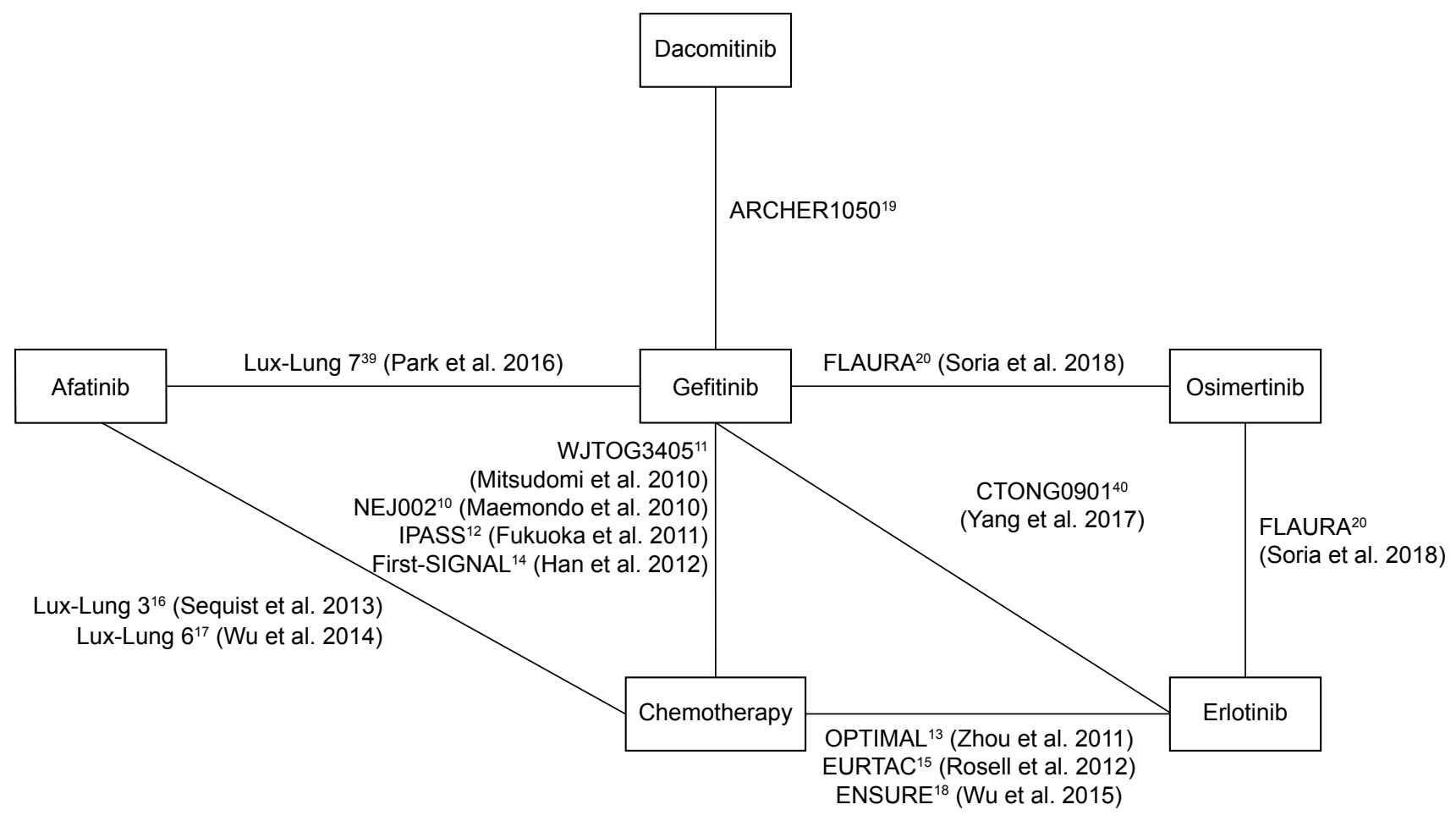

Figure 2 Complete network based on 13 RCTs.

Abbreviation: RCT, randomized controlled trial. 
Table 3 Treatment comparisons for PFS, OS (HRs [95\% Cl]), ORR, diarrhea, and rash (ORs [95\% Cl])

\begin{tabular}{|c|c|c|c|c|c|}
\hline $\begin{array}{l}\text { PFS } \\
\text { Chemotherapy } \\
0.43(0.37,0.49) \\
0.36(0.30,0.43) \\
0.37(0.31,0.44) \\
0.25(0.19,0.33) \\
0.18(0.14,0.22)\end{array}$ & $\begin{array}{l}2.34(2.03,2.7 \mathrm{I}) \\
\text { Gefitinib } \\
0.85(0.7 \mathrm{I}, \mathrm{I} .02) \\
0.87(0.72,1.04) \\
0.59(0.47,0.74) \\
0.42(0.34,0.5)\end{array}$ & $\begin{array}{l}2.76(2.3,3.34) \\
1.17(0.98,1.4 \mathrm{I}) \\
\text { Erlotinib } \\
1.03(0.8,1.3) \\
0.7(0.52,0.93) \\
0.49(0.41,0.59)\end{array}$ & $\begin{array}{l}2.7(2.27,3.24) \\
1.15(0.96,1.39) \\
0.97(0.77,1.25) \\
\text { Afatinib } \\
0.69(0.51,0.91) \\
0.48(0.37,0.62)\end{array}$ & $\begin{array}{l}3.95(3.05,5.21) \\
1.68(1.35,2.13) \\
1.42(1.08,1.93) \\
1.45(1.09,1.97) \\
\text { Dacomitinib } \\
0.71(0.52,0.94)\end{array}$ & $\begin{array}{l}5.64(4.58,7.02) \\
2.4(2,2.91) \\
2.04(1.7,2.46) \\
2.07(1.62,2.69) \\
1.41(1.06,1.91) \\
\text { Osimertinib }\end{array}$ \\
\hline $\begin{array}{l}\text { OS } \\
\text { Chemotherapy } \\
1.03(0.89,1.19) \\
1.01(0.84,1.21) \\
0.9(0.76,1.06) \\
0.79(0.58,1.06) \\
0.65(0.49,0.84)\end{array}$ & $\begin{array}{l}0.97(0.84, I .12) \\
\text { Gefitinib } \\
0.98(0.80,1.19) \\
0.88(0.73,1.05) \\
0.77(0.58,0.99) \\
0.63(0.48,0.81)\end{array}$ & $\begin{array}{l}0.99(0.83,1.19) \\
1.02(0.84,1.24) \\
\text { Erlotinib } \\
0.90(0.71,1.13) \\
0.79(0.56,1.08) \\
0.64(0.49,0.82)\end{array}$ & $\begin{array}{l}I . I I(0.94, I .31) \\
I . I 4(0.96, I .38) \\
I . I I(0.89, I .42) \\
\text { Afatinib } \\
0.88(0.63, I .2 I) \\
0.72(0.53,0.96)\end{array}$ & $\begin{array}{l}1.26(0.94,1.73) \\
1.3(1.01,1.72) \\
1.27(0.93,1.8) \\
\text { I.I3 (0.83, I.59) } \\
\text { Dacomitinib } \\
0.84(0.57,1.19)\end{array}$ & $\begin{array}{l}1.54(1.19,2.04) \\
1.59(1.24,2.07) \\
1.56(1.22,2.03) \\
1.38(1.04,1.89) \\
1.20(0.84,1.77) \\
\text { Osimertinib }\end{array}$ \\
\hline $\begin{array}{l}\text { ORR } \\
\text { Chemotherapy } \\
3.86(2.94,5) \\
5.09(3.66,6.91) \\
6.08(4.45,8.1) \\
4.60(3.23,6.37) \\
6.18(3.65,9.8)\end{array}$ & $\begin{array}{l}0.26(0.2,0.34) \\
\text { Gefitinib } \\
1.33(0.93,1.86) \\
1.59(1.13,2.17) \\
1.19(0.96,1.46) \\
1.61(0.98,2.49)\end{array}$ & $\begin{array}{l}0.20(0.14,0.27) \\
0.75(0.54,1.08) \\
\text { Erlotinib } \\
1.22(0.79,1.81) \\
0.92(0.6,1.35) \\
1.23(0.75,1.9)\end{array}$ & $\begin{array}{l}0.16(0.12,0.22) \\
0.63(0.46,0.89) \\
0.82(0.55,1.27) \\
\text { Afatinib } \\
0.77(0.51,1.12) \\
1.04(0.58,1.72)\end{array}$ & $\begin{array}{l}0.22(0.16,0.31) \\
0.84(0.68,1.04) \\
1.08(0.74,1.66) \\
1.30(0.9,1.96) \\
\text { Dacomitinib } \\
1.37(0.79,2.2)\end{array}$ & $\begin{array}{l}0.16(0.10,0.27) \\
0.62(0.4,1.02) \\
0.82(0.53,1.34) \\
0.97(0.58,1.73) \\
0.73(0.45,1.26) \\
\text { Osimertinib }\end{array}$ \\
\hline $\begin{array}{l}\text { Diarrhea } \\
\text { Chemotherapy } \\
4(0.74, \mid 2.8 I) \\
5.11(0.76,|7.9|) \\
39.8(6.7 I,|3| .4) \\
53.34(3.96,239.4) \\
4.58(0.69,15.86)\end{array}$ & $\begin{array}{l}0.25(0.08,1.36) \\
\text { Gefitinib } \\
1.55(0.3,4.82) \\
12.01(2.7,35.35) \\
13.36(2.33,44) \\
1.26(0.37,3.16)\end{array}$ & $\begin{array}{l}0.2(0.06, \mid \mathrm{I} .32) \\
0.65(0.2 \mathrm{I}, 3.37) \\
\text { Erlotinib } \\
\mathrm{I} 2.03(\mathrm{I} .38,46.74) \\
\mathrm{I} 4.36(\mathrm{I} . \mathrm{I}, 64.27) \\
\mathrm{I} .05(0.3 \mathrm{I}, 2.63)\end{array}$ & $\begin{array}{l}0.03(0.01,0.15) \\
0.08(0.03,0.37) \\
0.08(0.02,0.73) \\
\text { Afatinib } \\
1.71(0.15,7.28) \\
0.16(0.02,0.55)\end{array}$ & $\begin{array}{l}0.02(0,0.25) \\
0.07(0.02,0.43) \\
0.07(0.02,0.91) \\
0.59(0.14,6.84) \\
\text { Dacomitinib } \\
0.17(0.02,0.66)\end{array}$ & $\begin{array}{l}0.22(0.06, I .45) \\
0.79(0.32,2.67) \\
0.95(0.38,3.2 \mathrm{I}) \\
6.39(\mathrm{I} .82,43.4) \\
6.04(\mathrm{I} .52,58.3 \mathrm{I}) \\
\text { Osimertinib }\end{array}$ \\
\hline $\begin{array}{l}\text { Rash } \\
\text { Chemotherapy } \\
4.28(1.4,10.23) \\
9.18(2.39,24.56) \\
18.06(4.51,49.39) \\
1,170(19.4,7,267) \\
12.94(2.87,37.81)\end{array}$ & $\begin{array}{l}0.23(0.1,0.7 I) \\
\text { Gefitinib } \\
2.46(0.63,6.74) \\
4.47(1.54,10.17) \\
275.4(6.1,1,636) \\
3.24(0.92,8.28)\end{array}$ & $\begin{array}{l}0.1 I(0.04,0.42) \\
0.4 I(0.15,1.6) \\
\text { Erlotinib } \\
2.57(0.45,8.35) \\
162(2.34, I .009) \\
1.59(0.46,4.03)\end{array}$ & $\begin{array}{l}0.06(0.02,0.22) \\
0.22(0.10,0.65) \\
0.39(0.12,2.21) \\
\text { Afatinib } \\
77.97(1.31,477.4) \\
0.9(0.17,2.83)\end{array}$ & $\begin{array}{l}0(0,0.05) \\
0(0,0.16) \\
0.01(0,0.43) \\
0.01(0,0.77) \\
\text { Dacomitinib } \\
0.09(0,0.55)\end{array}$ & $\begin{array}{l}0.08(0.03,0.35) \\
0.31(0.12,1.08) \\
0.63(0.25,2.18) \\
\text { I.II }(0.35,5.86) \\
\text { II.I5 }(1.82,740.19) \\
\text { Osimertinib }\end{array}$ \\
\hline
\end{tabular}

Abbreviations: OS, overall survival; PFS, progression-free survival; ORR, objective response rate.

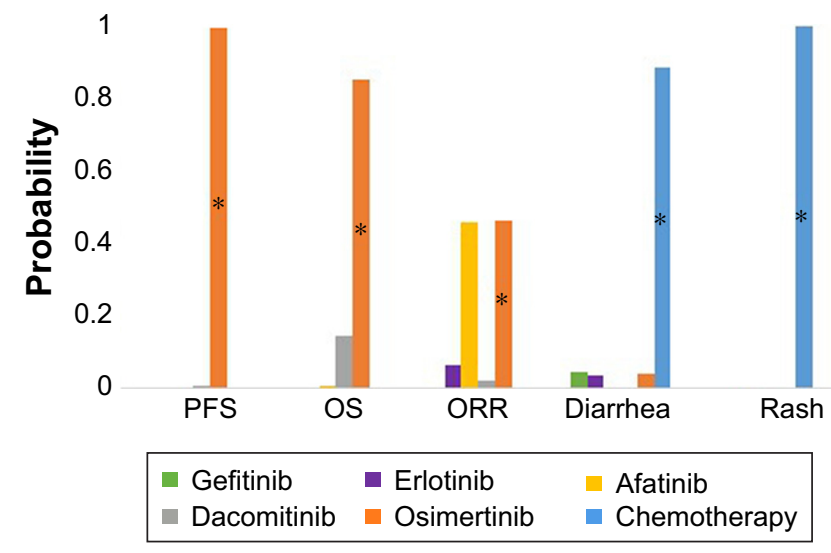

Figure 3 Distribution of probabilities of being the best for outcomes and two major toxicities, classified by drugs.

Note: $* P<0.0001$.

Abbreviations: ORR, objective response rate; OS, overall survival; PFS, progressionfree survival. differences between EGFR-TKIs in terms of efficacy and toxicity. Since a number of head-to-head trials between these drugs and data from new EGFR-TKIs are now available, we performed an NMA with five different TKIs (afatinib, dacomitinib, erlotinib, gefitinib, and osimertinib) to estimate their relative efficacy and toxicity as first-line treatment in patients with EGFR-mutated (exon 19 deletion or exon 21 L858R mutation) NSCLC. The results of the NMA indicated that osimertinib was significantly more effective on PFS compared to all other drugs. Dacomitinib proved to be the second best TKI effective on PFS with a significantly better PFS compared to gefitinib, erlotinib, and afatinib. Osimertinib also showed a significantly better efficacy in terms of OS compared to all other TKIs. Furthermore, AEs (diarrhea and rash) occurred more often in patients treated with afatinib 
or dacomitinib, compared to the other treatments. Due to the limited number of trials per treatment arm, a fixed-effect NMA was considered appropriate because heterogeneity could not be appropriately assessed. ${ }^{33-35}$

To our knowledge, this is the first study that performed an NMA to compare the results between five first-line EGFRTKIs. Previous NMA studies failed to show significant differences between EGFR-TKIs. ${ }^{22-26}$ By including additional evidence from new RCTs ${ }^{19,20,40}$ and updating results in the network, new results were produced, namely significant efficacy differences between the TKIs.

An important assumption in our study was that all included studies were generally similar, both clinically and methodologically. All 13 studies included only patients with activating EGFR mutations, with the percentage of males ranging from $11 \%$ to $47 \%$, the median age range being 56-65 years, and the percentage of adenocarcinoma histology type ranging between $90 \%$ and $100 \%$ across the studies, which contributed to the homogeneity of the study population. Additionally, efficacy of EGFR-TKIs could be different when it was provided as second- or third-line treatment. A previous study showed that chemotherapy might change the proportion of tumor cells with EGFR mutations within the primary tumor. ${ }^{45}$ Treatment with a TKI after platinum-based doublet therapy would thus probably affect the efficacy by inducing resistance mechanisms. Therefore, only first-line TKI treatments were included in our analyses in order to avoid such bias and to improve homogeneity.

For our analysis, the most common NMA method was used and, consequently, proportional hazards were assumed. ${ }^{32}$ Since in eleven of the 13 trials the proportional hazard assumption could be checked, the assumption was not violated.

The length of follow-up differed among the included studies. As HRs may depend on the follow-up period, findings may vary when HRs are estimated at a different followup period. Due to a lack of patient-level data, correction for the different lengths of follow-up in an NMA is not possible. Insight into the long-term direction of HRs can be obtained with a longer follow-up duration, although this will also induce selection bias. ${ }^{46}$

Although osimertinib showed a significant better OS compared to all drugs, gefitinib, erlotinib, and afatinib did not reveal a significant effect on OS compared to chemotherapy, which was similar to the individual studies. Some individual studies even showed OS results which were in favor of chemotherapy due to high proportions of crossover in the chemotherapy arms. ${ }^{9,11,13-15}$ The minimum proportion of crossover in the chemotherapy arm was $59.3 \%$ in the WJTOG3405 study ${ }^{11}$ and the maximum was $94.6 \%$ in the NEJ002 study. ${ }^{10}$ A much smaller proportion of patients in whom TKI was initiated received chemotherapy as subsequent treatment. ${ }^{10,11,13-17} \mathrm{~A}$ recent study suggested that patients who received chemotherapy or TKI after first-line TKI or first-line chemotherapy had a longer OS than patients who only received first-line therapy. ${ }^{42}$ The imbalanced subsequent treatments of the TKI and chemotherapy arms may have resulted in no significant OS differences between TKIs and chemotherapy. Therefore, it is questionable whether OS is an appropriate outcome measure in studies with substantial crossover.

Since final OS data were not available during our study period, the OS data of the FLAURA study were based on an interim analysis. Although this analysis did not show a formal statistical significance for OS, osimertinib seems to show a potential survival benefit compared to standard TKI. ${ }^{20}$ An update of our NMA is desirable when final OS data of the FLAURA trial become available.

\section{Conclusion}

Our study showed that osimertinib is the most favorable EGFR-TKI in terms of PFS and OS. With regard to AEs, afatinib and dacomitinib had a higher risk of diarrhea and rash. Gefitinib, erlotinib, and osimertinib showed a mild risk of AEs. Thus, regarding its high efficacy and mild toxicity pattern, osimertinib is indicated as the most favorable firstline TKI in patients with activating EGFR-mutated (exon 19 deletion or exon 21 L858R mutation) NSCLC.

\section{Disclosure}

Carin A Uyl-de Groot reports grants received from Boehringer-Ingelheim, Janssen-Cilag B.V., Genzyme, Astellas Pharma B.V., Sanofi Netherlands, Roche Netherlands B.V., AstraZeneca Netherlands, Amgen Europe B.V., Gilead Sciences, Merck, and Bayer, outside the submitted work. The other authors report no conflicts of interest in this work.

\section{References}

1. World Health Organization. Fact sheet cancer. Available from: http://www.who.int/mediacentre/factsheets/fs297/en/. Accessed September, 2017.

2. Sun S, Schiller JH, Spinola M, Minna JD. New molecularly targeted therapies for lung cancer. J Clin Invest. 2007;117(10):2740-2750.

3. Alvarez M, Roman E, Santos ES, Raez LE. New targets for non-smallcell lung cancer therapy. Expert Rev Anticancer Ther. 2007;7(10): 1423-1437.

4. Kerner GS, Schuuring E, Sietsma J, et al. Common and rare EGFR and KRAS mutations in a Dutch non-small-cell lung cancer population and their clinical outcome. PLoS One. 2013;8(7):e70346.

5. Zhang YL, Yuan JQ, Wang KF, et al. The prevalence of EGFR mutation in patients with non-small cell lung cancer: a systematic review and meta-analysis. Oncotarget. 2016;7(48):78985-78993. 
6. Lynch TJ, Bell DW, Sordella R, et al. Activating mutations in the epidermal growth factor receptor underlying responsiveness of Non-SmallCell lung cancer to gefitinib. NEngl J Med.2004;350(21):2129-2139.

7. Paez JG. EGFR mutations in lung cancer: correlation with clinical response to gefitinib therapy. Science. 2004;304(5676):1497-1500.

8. Pao W, Miller V, Zakowski M, et al. EGF receptor gene mutations are common in lung cancers from "never smokers" and are associated with sensitivity of tumors to gefitinib and erlotinib. Proc Natl Acad Sci U S A. 2004;101(36):13306-13311.

9. Mok TS, Wu YL, Thongprasert S, et al. Gefitinib or carboplatinpaclitaxel in pulmonary adenocarcinoma. N Engl J Med. 2009; 361(10):947-957.

10. Maemondo M, Inoue A, Kobayashi K, et al. Gefitinib or chemotherapy for non-small-cell lung cancer with mutated EGFR. $N$ Engl $J$ Med. 2010;362(25):2380-2388

11. Mitsudomi T, Morita S, Yatabe Y, et al. Gefitinib versus cisplatin plus docetaxel in patients with non-small-cell lung cancer harbouring mutations of the epidermal growth factor receptor (WJTOG3405): an open label, randomised phase 3 trial. Lancet Oncol. 2010;11(2):121-128.

12. Fukuoka M, Wu YL, Thongprasert S, et al. Biomarker analyses and final overall survival results from a phase III, randomized, open-label, first-line study of gefitinib versus carboplatin/paclitaxel in clinically selected patients with advanced non-small-cell lung cancer in Asia (IPASS). J Clin Oncol. 2011;29(21):2866-2874.

13. Zhou C, Wu Y-L, Chen G, et al. Erlotinib versus chemotherapy as first-line treatment for patients with advanced EGFR mutation-positive non-small-cell lung cancer (optimal, CTONG-0802): a multicentre, open-label, randomised, phase 3 study. Lancet Oncol. 2011;12(8): 735-742.

14. Han J-Y, Park K, Kim S-W, et al. First-SIGNAL: first-line single-agent Iressa versus gemcitabine and cisplatin trial in never-smokers with adenocarcinoma of the lung. J Clin Oncol. 2012;30(10):1122-1128.

15. Rosell R, Carcereny E, Gervais R, et al. Erlotinib versus standard chemotherapy as first-line treatment for European patients with advanced EGFR mutation-positive non-small-cell lung cancer (EURTAC): a multicentre, open-label, randomised phase 3 trial. Lancet Oncol. 2012;13(3):239-246.

16. Sequist LV, Yang JC, Yamamoto N, et al. Phase III study of afatinib or cisplatin plus pemetrexed in patients with metastatic lung adenocarcinoma with EGFR mutations. J Clin Oncol. 2013;31(27): 3327-3334

17. Wu Y-L, Zhou C, Hu C-P, et al. Afatinib versus cisplatin plus gemcitabine for first-line treatment of Asian patients with advanced nonsmall-cell lung cancer harbouring EGFR mutations (LUX-Lung 6): an open-label, randomised phase 3 trial. Lancet Oncol. 2014;15(2): 213-222.

18. Wu YL, Zhou C, Liam CK, et al. First-line erlotinib versus gemcitabine/ cisplatin in patients with advanced EGFR mutation-positive non-smallcell lung cancer: analyses from the phase III, randomized, open-label, ensure study. Ann Oncol. 2015;26(9):1883-1889.

19. Wu YL, Cheng Y, Zhou X, et al. Dacomitinib versus gefitinib as firstline treatment for patients with EGFR-mutation-positive non-small-cell lung cancer (Archer 1050): a randomised, open-label, phase 3 trial. Lancet Oncol. 2017;18(11):1454-1466.

20. Soria J-C, Ohe Y, Vansteenkiste J, et al. Osimertinib in untreated $E G F R$-mutated advanced non-small-cell lung cancer. $N$ Engl J Med. 2018;378(2):113-125.

21. Jansen JP, Fleurence R, Devine B, et al. Interpreting indirect treatment comparisons and network meta-analysis for health-care decision making: report of the ISPOR Task Force on indirect treatment comparisons good research practices: Part 1. Value Health. 2011;14(4):417-428.

22. Liang W, Wu X, Fang W, et al. Network meta-analysis of erlotinib, gefitinib, afatinib and icotinib in patients with advanced non-small-cell lung cancer harboring EGFR mutations. PLoS One. 2014;9(2):e85245.

23. Popat S, Mok T, Yang JC-H, et al. Afatinib in the treatment of EGFR mutation-positive NSCLC - a network meta-analysis. Lung Cancer. 2014;85(2):230-238.
24. Zhang Y, Sheng J, Yang Y, et al. Optimized selection of three major EGFR-TKIs in advanced EGFR-positive non-small cell lung cancer: a network meta-analysis. Oncotarget. 2016;7(15):20093-20108.

25. Batson S, Mitchell SA, Windisch R, Damonte E, Munk V, Reguart N. Tyrosine kinase inhibitor combination therapy in first-line treatment of non-small-cell lung cancer: systematic review and network metaanalysis. OncoTargets and Therapy. 2017;10(10):2473-2482.

26. Zhang Y, Zhang Z, Huang X, et al. Therapeutic efficacy comparison of 5 major EGFR-TKIs in advanced EGFR-positive Non-Small-cell lung cancer: a network meta-analysis based on head-to-head trials. Clin Lung Cancer. 2017;18(5):e333-e340.

27. Moher D, Liberati A, Tetzlaff J, Altman DG; The PRISMA Group. Preferred reporting items for systematic reviews and meta-analyses: the PRISMA statement. PLoS Med. 2009;6(7):e1000097.

28. U.S. Department of Health and Human Services. Common terminology criteria for adverse events (CTCAE) version 5.0. Bethesda, MD: National Cancer Institute. 2017

29. Califano R, Tariq N, Compton S, et al. Expert consensus on the management of adverse events from EGFR tyrosine kinase inhibitors in the UK. Drugs. 2015;75(12):1335-1348.

30. Hirsh V. Managing treatment-related adverse events associated with EGFR tyrosine kinase inhibitors in advanced non-small-cell lung cancer. Current Oncology. 2011;18(3):126-138.

31. Higgins JPT, Altman DG, Gotzsche PC, et al. The Cochrane collaboration's tool for assessing risk of bias in randomised trials. BMJ. 2011; 343(2):d5928.

32. Dias S, Welton NJ, Sutton AJ, Ades AE. A generalized linear modelling framework for pairwise and network meta-analysis of randomized controlled trials. Sheffield: ScHARR, University of Sheffield. 2011:1-98.

33. van Beurden-Tan CHY, Franken MG, Blommestein HM, Uyl-de Groot CA, Sonneveld P. Systematic literature review and network metaanalysis of treatment outcomes in relapsed and/or refractory multiple myeloma. J Clin Oncol. 2017;35(12):1312-1319.

34. Weisel K, Doyen C, Dimopoulos M, et al. A systematic literature review and network meta-analysis of treatments for patients with untreated multiple myeloma not eligible for stem cell transplantation. Leuk Lymphoma. 2017;58(1):153-161.

35. Higgins JPT, Green S. Cochrane Handbook for Systematic Reviews of Interventions Version 5.1.0 [updated March 2011]. London: The Cochrane Collaboration; 2011.

36. Brooks SP, Gelman A. General methods for monitoring convergence of iterative simulations. J Comput Graph Stat. 1998;7:434-455.

37. Inoue A, Kobayashi K, Maemondo M, et al. Updated overall survival results from a randomized phase III trial comparing gefitinib with carboplatin-paclitaxel for chemo-naïve non-small cell lung cancer with sensitive EGFR gene mutations (NEJ002). Ann Oncol. 2013;24(1):54-59.

38. Yoshioka H, Mitsudomi T, Morita S, et al. Final overall survival results of WJTOG 3405, a randomized phase 3 trial comparing gefitinib (G) with cisplatin plus docetaxel (CD) as the first-line treatment for patients with non-small cell lung cancer (NSCLC) harboring mutations of the epidermal growth factor receptor (EGFR). J Clin Oncol. 2014; 32(15_suppl):8117.

39. Park K, Tan E-H, O'Byrne K, et al. Afatinib versus gefitinib as first-line treatment of patients with $E G F R$ mutation-positive non-small-cell lung cancer (LUX-Lung 7): a phase 2B, open-label, randomised controlled trial. Lancet Oncol. 2016;17(5):577-589.

40. Yang JJ, Zhou Q, Yan HH, et al. A phase III randomised controlled trial of erlotinib vs gefitinib in advanced non-small cell lung cancer with EGFR mutations. Br J Cancer. 2017;116(5):568-574.

41. Mok T, Cheng Y, Zhou X, et al. Dacomitinib (daco) versus gefitinib (GEF) for first-line treatment of advanced NSCLC (Archer 1050): final overall survival (OS) analysis. J Clin Oncol. 2018;36(15_suppl): 9004.

42. Zhou C, Wu YL, Chen G, et al. Final overall survival results from a randomised, phase III study of erlotinib versus chemotherapy as firstline treatment of EGFR mutation-positive advanced non-small-cell lung cancer (optimal, CTONG-0802). Ann Oncol. 2015;26(9):1877-1883. 
43. Yang JC-H, Wu Y-L, Schuler M, et al. Afatinib versus cisplatin-based chemotherapy for EGFR mutation-positive lung adenocarcinoma (LUX-Lung 3 and LUX-Lung 6): analysis of overall survival data from two randomised, phase 3 trials. Lancet Oncol. 2015;16(2):141-151.

44. Ingelheim B. Overall survival data from LUX-Lung 7 head-to-head trial of afatinib versus gefitinib presented at ESMO 2016. Available from: https:/www.boehringer-ingelheim.com/press-release/overallsurvival-data-lux-lung-7-head-head-trial2016. Accessed April, 2017.
45. Bai $\mathrm{H}$, Wang $\mathrm{Z}$, Chen $\mathrm{K}$, et al. Influence of chemotherapy on EGFR mutation status among patients with non-small-cell lung cancer. J Clin Oncol. 2012;30(25):3077-3083.

46. Hernán MA. The hazards of hazard ratios. Epidemiology. 2010;21(1): $13-15$.

\section{Publish your work in this journal}

OncoTargets and Therapy is an international, peer-reviewed, open access journal focusing on the pathological basis of all cancers, potential targets for therapy and treatment protocols employed to improve the management of cancer patients. The journal also focuses on the impact of management programs and new therapeutic agents and protocols on

\section{Dovepress}

patient perspectives such as quality of life, adherence and satisfaction. The manuscript management system is completely online and includes a very quick and fair peer-review system, which is all easy to use. Visit http://www.dovepress.com/testimonials.php to read real quotes from published authors.

Submit your manuscript here: http://www.dovepress.com/oncotargets-and-therapy-journal 